\title{
PSMA Ligands for PET Imaging of Prostate Cancer
}

\author{
Sarah M. Schwarzenboeck ${ }^{1}$, Isabel Rauscher ${ }^{2}$, Christina Bluemel ${ }^{3}$, Wolfgang P. Fendler ${ }^{4,5}$, Steven P. Rowe ${ }^{6}$, \\ Martin G. Pomper 6 , Ali Asfhar-Oromieh ${ }^{7,8}$, Ken Herrmann ${ }^{4,9}$, and Matthias Eiber ${ }^{2,4}$ \\ ${ }^{I}$ Department of Nuclear Medicine, Rostock University Medical Centre, Rostock, Germany; ${ }^{2}$ Department of Nuclear Medicine, \\ Klinikum rechts der Isar, Technical University of Munich, Munich, Germany; ${ }^{3}$ Department of Nuclear Medicine, University Hospital \\ Würzburg, Würzburg, Germany; ${ }^{4}$ Department of Molecular and Medical Pharmacology, David Geffen School of Medicine at UCLA, \\ Los Angeles, California; ${ }^{5}$ Department of Nuclear Medicine, Ludwig-Maximilians-University of Munich, Munich, Germany; ${ }^{6}$ Russell \\ H. Morgan Department of Radiology and Radiological Science, Johns Hopkins University School of Medicine, Baltimore, Maryland; \\ ${ }^{7}$ Department of Nuclear Medicine, Heidelberg University Hospital, Heidelberg, Germany; ${ }^{8}$ Clinical Cooperation Unit Nuclear \\ Medicine, German Cancer Research Centre, Heidelberg, Germany; and ${ }^{9}$ Klinik für Nuklearmedizin, Universitätsklinikum Essen, \\ Essen, Germany
}

Learning Objectives: On successful completion of this activity, participants should be able to (1) recognize the current status of PSMA ligand PET imaging (clinical indications, diagnostic value, impact on treatment planning), (2) apply PSMA PET/CT (patient preparation, image acquisition), and (3) interpret PSMA imaging.

Financial Disclosure: Martin G. Pomper is a coinventor on a U.S. Patent covering ${ }^{18} \mathrm{~F}-\mathrm{DCFPyL}$, and as such is entitled to a portion of any licensing fees and royalties generated by this technology. This arrangement has been reviewed and approved by the Johns Hopkins University in accordance with its conflict-ofinterest policies. Martin G. Pomper and Steven P. Rowe have received research support from Progenics Pharmaceuticals, the licensee of ${ }^{18} \mathrm{~F}-\mathrm{DCFP}$. $\mathrm{L}$. The authors of this article have indicated no other relevant relationships that could be perceived as a real or apparent conflict of interest.

CME Credit: SNMMI is accredited by the Accreditation Council for Continuing Medical Education (ACCME) to sponsor continuing education for physicians. SNMMI designates each JNM continuing education article for a maximum of 2.0 AMA PRA category 1 credits. Physicians should claim only credit commensurate with the extent of their participation in the activity. For CE credit, SAM, and other credit types, participants can access this activity through the SNMMI website (http://www.snmmilearningcenter.org) through Xxxxxx 2020.

Targeting the prostate-specific membrane antigen (PSMA) with ${ }^{68} \mathrm{Ga}$-labeled and ${ }^{18} \mathrm{~F}$-labeled PET agents has become increasingly important in recent years. Imaging of biochemically recurrent prostate cancer has been established as a widely accepted clinical indication for PSMA ligand PET/CT in many parts of the world because of the results of multiple, primarily retrospective, studies that indicate superior detection efficacy compared with standard-of-care imaging. For high-risk primary prostate cancer, evidence is growing that this modality significantly aids in the detection of otherwise occult nodal and bone metastases. For both clinical indications in recurrent as well as in primary prostate cancer, preliminary data demonstrate a substantial impact on clinical management. Emerging data imply that intraprostatic tumor localization, therapy stratification, and treatment monitoring of advanced disease in specific clinical situations might become future indications. Current criteria for image reporting of PSMA ligand PET are evolving given the expanding body of literature on physiologic and pathologic uptake patterns and pitfalls. This article intends to give an educational overview on the current status of PSMA ligand PET imaging, including imaging procedure and interpretation, clinical indications, diagnostic potential, and impact on treatment planning.

Key Words: prostate cancer; PET/CT; PSMA

J Nucl Med 2017; 58:1545-1552

DOI: 10.2967/jnumed.117.191031

Received May 26, 2017; revision accepted Jul. 6, 2017.

For correspondence or reprints contact: Sarah M. Schwarzenböck, Department of Nuclear Medicine, Rostock University Medical Centre, Gertrudenplatz 1, 18057 Rostock, Germany.

E-mail: sarah.schwarzenboeck@med.uni-rostock.de

Published online Jul. 7, 2017.

COPYRIGHT (c) 2017 by the Society of Nuclear Medicine and Molecular Imaging.
$\mathbf{P}$ ostate cancer (PC) is the most common cancer in men and the third most frequent cause of cancer-related death in men worldwide (1). After primary treatment, biochemical recurrence (BCR) occurs in approximately $30 \%-40 \%$ of patients. After potential salvage treatment options, patients are usually treated with androgendeprivation therapy (ADT). Typically, after 2-8 y of ADT, prostatespecific antigen (PSA) begins to rise again, indicating metastatic castration-resistant PC, the lethal form of the disease.

In the primary setting, detection of extraprostatic spread is crucial for further treatment planning and determination of prognosis. However, the cross-sectional imaging and bone scintigraphy recommended in many guidelines have shown limitations in detecting sites of nodal or bone involvement in preoperative patients $(2-4)$. Further, in patients with high suspicion of PC, multiparametric MRI helps to rule out clinically significant disease and to guide targeted biopsy (5), although multiparametric MRI can miss aggressive PC lesions (6). In BCR, accurate restaging is crucial because local versus systemic disease substantially influences further treatment management. Accurate diagnosis of the site and extent of disease can be used in tailoring potential salvage treatments; however, standardof-care imaging also has sensitivity and specificity limitations in this regard.

In contrast, the use of PET/CT, combining functional and morphologic information, for $\mathrm{PC}$ imaging has been increasing within the last decade. ${ }^{18} \mathrm{~F}-\mathrm{FDG}$ is the most widely used radiotracer in oncologic PET/CT imaging; however, only a minority of PC (i.e., only aggressive, poorly differentiated, or undifferentiated PC) shows a high glycolytic rate, limiting the use of ${ }^{18} \mathrm{~F}-\mathrm{FDG} \operatorname{PET}(7,8)$. In Europe, radiolabeled choline derivatives $\left({ }^{18} \mathrm{~F}\right.$-fluorocholine or ${ }^{11}$ C-choline) were among the most commonly used PET tracers for PC imaging. They were most frequently used for restaging of PC 
and primary staging in selected cases (e.g., high-risk PC). Detection and localization of primary PC are limited by nonspecific uptake in benign intraprostatic pathologies (9). Recent metaanalyses reported a high specificity of $95 \%$ but a poor sensitivity of $49 \%$ in primary nodal staging (10). Detection rates are positively associated with PSA level but are low $(<50 \%)$ in patients with early BCR (i.e., PSA $<2 \mathrm{ng} / \mathrm{mL}$ ) (11). Other PET radiopharmaceuticals have been investigated (e.g., ${ }^{11} \mathrm{C}$-acetate) or even Food and Drug Administrationapproved (e.g., ${ }^{18} \mathrm{~F}$-fluciclovine), in part demonstrating superiority over choline derivatives (12-15).

Given the limitations of the most widely investigated PET tracers, targeting the prostate-specific membrane antigen (PSMA) with molecular imaging agents has recently been increasingly investigated. PSMA is a transmembrane protein that is highly overexpressed (100- to 1,000-fold) on almost all PC tumors (1619). Only $5 \%-10 \%$ of primary PC lesions have been shown to be PSMA-negative $(20,21)$. PSMA expression levels increase with higher tumor stage and grade $(16,18,22)$. Presently, the only Food and Drug Administration-approved PSMA agent is a radiolabeled anti-PSMA antibody (ProstaScint, capromab pendetide; EUSA Pharma); however, this targets an intracellular epitope of PSMA (7E11) (19) that cannot be accessed in viable tumor cells, limiting diagnostic performance (23).

In contrast, small-molecule PSMA ligands bind to the active site in the extracellular domain of PSMA and are internalized and endosomally recycled, leading to enhanced tumor uptake and retention and high image quality (24-27). The most widely used ${ }^{68} \mathrm{Ga}$-labeled PSMA ligands for PET imaging are ${ }^{68} \mathrm{Ga}$-PSMA-11 $\left({ }^{68} \mathrm{Ga}-\mathrm{PSMA}-\mathrm{HBED}-\mathrm{CC}\right)$ and the theranostic agents ${ }^{68} \mathrm{Ga}$-PSMA617 and ${ }^{68} \mathrm{Ga}-\mathrm{PSMA}-\mathrm{I} \& \mathrm{~T}(28,29) .{ }^{18} \mathrm{~F}$-labeled agents include ${ }^{18}$ F-DCFBC (30,31), ${ }^{18} \mathrm{~F}-D C F P y L ~(32)$, and ${ }^{18}$ F-PSMA 1007 (33). They exploit the average lower positron range (reducing blurring effects), longer half-life, and potential for centralized production and distribution of ${ }^{18} \mathrm{~F}$ compared with ${ }^{68} \mathrm{Ga}$. A tabular overview of the most common PSMA ligands in clinical use was recently published (34).

This article intends to give an educational overview on the current status of PSMA ligand PET, including imaging procedure and interpretation, clinical indications, diagnostic potential, and impact on treatment planning.

\section{MAIN CLINICAL INDICATIONS OF PSMA LIGAND PET/CT AND CURRENT EVIDENCE IN THE LITERATURE}

\section{Biochemical Recurrence}

Approximately $30 \%-40 \%$ of patients will fail primary treatment, with a rising PSA level indicating recurrent or metastatic disease. Depending on the localization and extent of disease and prior treatment, different salvage options are available. Salvage surgery or salvage radiotherapy is used for local and nodal recurrence; stereotactic radiotherapy, for oligometastatic disease or systemic treatment in disseminated disease. Therefore, accurate restaging is crucial in recurrent PC patients. Currently, imaging of BCR is the most clinically accepted and validated indication for PSMA ligand PET/CT. Although a prospective head-to-head comparison of ${ }^{68} \mathrm{Ga}$-PSMA ligands and choline derivatives is missing, several, mainly retrospective, studies investigating BCR patients showed a higher diagnostic efficacy for PSMA ligand than for choline derivatives (35-37). $\mathrm{SUV}_{\max }$ and tumor-to-background ratios were superior for ${ }^{68}$ Ga-PSMA-11 compared with ${ }^{18} \mathrm{~F}$-fluorocholine (35), and ${ }^{68} \mathrm{Ga}$-PSMA-11 showed a higher detection rate than ${ }^{11} \mathrm{C}$-choline for lymph nodes as well as bone metastases (37). Positive findings exclusively detected by ${ }^{18} \mathrm{~F}$-fluorocholine PET/CT were rare (36). Three large retrospective studies (including 319, 248, and 1,007 patients, respectively) reported detection rates for ${ }^{68} \mathrm{Ga}$-PSMA-11 PET/CT in BCR of $88 \%$, almost $90 \%$, and $79.5 \%$, respectively (3840 ). In patients after curative treatment with a very low PSA level of less than $0.5 \mathrm{ng} / \mathrm{mL}$ the reported detection rate of PSMA ligand PET/CT ranged from $50 \%$ to $58 \%$ in different studies $(36,38-40)$. A recent study including only patients after prior radiotherapy (median PSA of $5.8 \mathrm{ng} / \mathrm{mL}$ ) presented detection rates of $33.3 \%$ for a PSA of less than $0.5 \mathrm{ng} / \mathrm{mL}, 71.4 \%$ for a PSA of 0.5 to less than 1 $\mathrm{ng} / \mathrm{mL}$, and $93.3 \%$ for a PSA of 1 to less than $2 \mathrm{ng} / \mathrm{mL}$. Local recurrence after radiotherapy was reported in $71 \%$ of the cohort, and $40 \%$ had suspected lymph node metastasis (41). A first metaregression analysis in a systematic review including 10 studies was recently published. It resulted in a predicted PSMA ligand PET/CT positivity rate of $42 \%, 58 \%, 76 \%$, and $95 \%$ for PSA values of $0-0.2,0.2-1,1-2$, and more than $2 \mathrm{ng} / \mathrm{mL}$, respectively (42). However, the results of this analysis need to be interpreted with caution as different ${ }^{68} \mathrm{Ga}$-PSMA-based PET tracers were pooled and no systematic histologic verification was available. Figures 1 and 2 show examples of nodal and local recurrence detected by ${ }^{68} \mathrm{Ga}$-PSMA ligand PET/CT.

Studies evaluating ${ }^{18} \mathrm{~F}$-labeled PSMA ligands suggest similar conclusions. In metastatic PC patients, the diagnostic performance of both ${ }^{18} \mathrm{~F}$-DCFBC and ${ }^{18} \mathrm{~F}$-DCFPyL PET/CT was superior to that of standard-of-care imaging for detecting suggestive lesions $(32,43)$. In a head-to-head comparison in 14 patients with recurrent $\mathrm{PC}$, staging with ${ }^{18} \mathrm{~F}-\mathrm{DCFPyL} \mathrm{PET} / \mathrm{CT}$ was equivalent to that with ${ }^{68}$ Ga-PSMA-11 (44). A follow-up study from the same group using PSA-adjusted parallel biochemically recurrent PC patient cohorts (including a total of 191 patients) found that ${ }^{18} \mathrm{~F}-\mathrm{DCFPyL}$ was noninferior to ${ }^{68} \mathrm{Ga}$-PSMA-11 (45) and suggested an improved sensitivity of the ${ }^{18} \mathrm{~F}$-labeled radiotracer in the PSA range of $0.5-3.5$ $\mathrm{ng} / \mathrm{mL}$ (with the caveat that different injected doses and acquisition parameters were used for the 2 PSMA-targeted agents). Further indepth clinical studies with standardized acquisition protocols and histologic validation are needed to establish the comparative performance of these 2 radiotracers.

\section{Primary Staging}

In high-risk PC patients, diagnosis of local extent and extraprostatic spread, that is, sites and extent of nodal and distant metastases, is crucial to further treatment planning (standard nodal dissection vs. extended dissection; change of primary radiotherapy field). Growing evidence underscores the role of PSMA ligand PET/CT imaging in primary PC, especially for N/M staging in a high-risk population. In detecting sites of nodal or bone involvement in preoperative patients, cross-sectional imaging has shown a limited pooled sensitivity and specificity of $42 \%$ and $82 \%$, respectively, for CT and $39 \%$ and $82 \%$, respectively, for MRI (3), as up to $80 \%$ of lymph node metastases in PC are harbored in normal-sized lymph nodes (2). Several studies showed a clear superiority of PSMA ligand PET/CT over standard-of-care imaging (CT, MRI, or bone scanning) (21,46-49). For example, in a retrospective analysis of 130 patients with primary intermediate- to high-risk PC using templatebased pelvic histopathology as a reference, ${ }^{68} \mathrm{Ga}$-PSMA-11 PET performed significantly better than morphologic imaging for $\mathrm{N}$ staging both on a patient and a template basis $(P=0.002$ and $<0.001$, respectively). On template-based analysis, the sensitivity, specificity, and accuracy were $68.3 \%, 99.1 \%$, and $95.2 \%$ for 

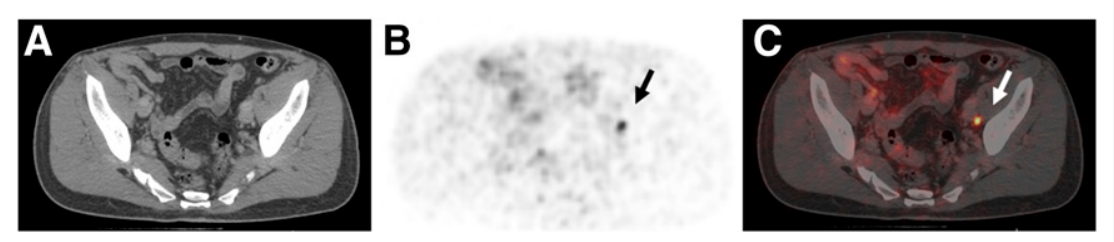

FIGURE 1. A 63-y-old patient with biochemical recurrence (PSA of $0.21 \mathrm{ng} / \mathrm{mL}$ ) after radical prostatectomy (initially pT2c N0 M0 L1/V1 R1 G1, Gleason score of 7), local radiation treatment, and antiandrogen therapy. ${ }^{68} \mathrm{Ga}-\mathrm{PSMA}$ ligand PET/CT exhibits solitary left iliac radiotracer-positive lymph node (arrow). Shown are transaxial CT (A), PET (B), and fused PET/CT (C) images. Patient was referred for salvage lymph node dissection.

${ }^{68}$ Ga-PSMA-11 PET and $27.3 \%, 97.1 \%$, and $87.6 \%$ for morphologic imaging, respectively (21). Similar results on the diagnostic efficacy of PSMA ligand PET for the detection of nodal metastases were obtained in other studies $(47,49)$. For bone metastases, Pyka et al. demonstrated that ${ }^{68} \mathrm{Ga}-\mathrm{PSMA}-11$ PET significantly outperformed bone scanning because of both its high sensitivity and its high specificity on a patient and region basis ( $P=0.006$ and $P<0.0001$, respectively). Because a histologic gold standard is not feasible in most cases for bone lesions, a best valuable comparator was defined on the basis of this study on a consensus review of all available current and follow-up images (including bone scanning/SPECT, PET, CT, MRI) and clinical data (48).

With regard to intraprostatic tumor localization by ${ }^{68} \mathrm{Ga}$-PSMA$11 \mathrm{PET} / \mathrm{CT}$, imaging findings were correlated with histopathology using segment- or voxel-based approaches in several studies (5052). These studies demonstrated relatively similar results, with a significantly higher ${ }^{68} \mathrm{Ga}$-PSMA-11 uptake in positive segments than in negative segments $\left(\mathrm{SUV}_{\max }\right.$ of 11.8 vs. 4.9 and $11.0 \mathrm{vs.}$ 2.7 , respectively, $P<0.001$ each) $(50,51)$. Results from combining ${ }^{68} \mathrm{Ga}$-PSMA-11 and multiparametric MRI on 53 preoperative intermediate-/high-risk patients indicated a potential for targeting biopsies. Hybrid ${ }^{68}$ Ga-PSMA-11 PET/MRI significantly outperformed multiparametric MRI and ${ }^{68} \mathrm{Ga}$-PSMA-11 PET in sensitivity and specificity for tumor localization on a sextant basis (respectively, $76 \%$ and $97 \%$ for hybrid ${ }^{68} \mathrm{Ga}$-PSMA-11 PET/MRI, $58 \%$ and $82 \%$ for multiparametric MRI, and $64 \%$ and $94 \%$ for ${ }^{68}$ Ga-PSMA PET) (53).

Using ${ }^{18} \mathrm{~F}$-labeled compounds in a first cohort of 13 patients, the sensitivity of MRI in the detection of primary PC was superior to that of ${ }^{18} \mathrm{~F}$-DCFBC PET/CT; however, ${ }^{18} \mathrm{~F}$-DCFBC PET/CT demonstrated a higher specificity for clinically significant disease (31). The relatively low sensitivity of ${ }^{18} \mathrm{~F}-\mathrm{DCFBC}$ in this context was likely at least partially attributable to its high blood-pool
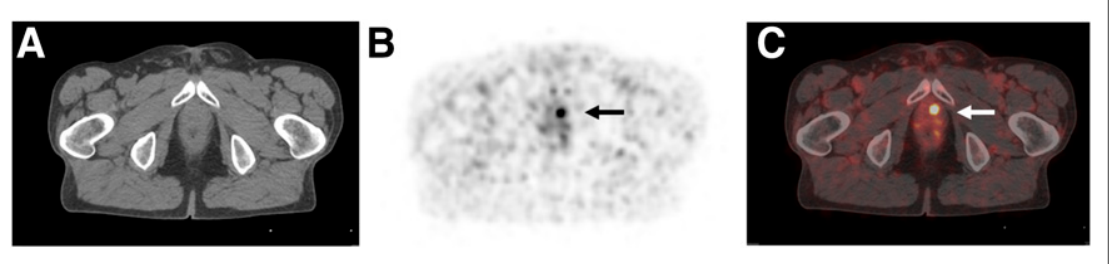

FIGURE 2. A 78-y-old patient with biochemical recurrence (PSA of $0.54 \mathrm{ng} / \mathrm{mL}$ ) after radical prostatectomy (initially pT3b NO MO R0 G2). ${ }^{68} \mathrm{Ga}$-PSMA ligand PET/CT reveals focal uptake in left paramedian prostatic fossa, indicating local recurrence. Shown are transaxial CT (A), PET (B), and fused PET/CT (C) images. Patient was referred for salvage radiation treatment. activity and low tumor-to-background ratios in relation to other small-molecule PSMA-targeted ligands, limitations potentially addressed by newer ${ }^{18} \mathrm{~F}$-labeled agents such as ${ }^{18} \mathrm{~F}$-DCFPyL and ${ }^{18} \mathrm{~F}$-PSMA 1007. A first retrospective study using ${ }^{18} \mathrm{~F}$ PSMA 1007 implied its high diagnostic potential by correctly detecting 18 of 19 histopathologically validated lymph node metastases in 8 patients with primary PC (33).

\section{Advanced Disease}

Typically, after 2-8 y of ADT the rise of PSA heralds the onset of metastatic castration-resistant PC, which is the lethal form of the disease and requires further systemic treatment (second ADT and taxane-based chemotherapy). The role of choline PET/CT in monitoring of systemic treatment in metastatic castration-resistant $\mathrm{PC}$ has been investigated in previously published studies and is still under debate $(54,55)$. Sclerotic bone metastases are not regarded as "target lesions" using RECIST 1.1, and bone scintigraphy suffers from the well-known flare phenomenon. As preclinical data show that changes in PSMA expression can indicate the therapeutic success of taxane-based therapy (56), PSMA ligand PET/CT might overcome many of the limitations of standard-of-care imaging. However, ADT might represent a potential confounder due to temporal PSMA upregulation after initiation, followed by downregulation and finally gross overexpression in androgen-resistant tumors as has been found in preliminary studies (57-60).

Monitoring systemic treatment in certain clinical scenarios may become a future indication for PSMA ligand imaging; however, evidence is currently still sparse (61). PSMA ligand PET/CT has an evolving role in PSMA-targeting treatments (e.g., radioligand therapy), evaluating target expression and therefore potentially predicting response (62-64). A rare but potential limitation is absent or low PSMA expression (e.g., in visceral metastases) in advanced disease, which may be related to therapy-induced specific biologic subtypes (e.g., neuroendocrine differentiated PC) $(65,66)$. Further information on the use of PSMA ligands for diagnosis has been published elsewhere $(67,68)$.

\section{IMPACT ON TREATMENT PLANNING}

Treatment management of PC is highly associated with the site and extent of disease (local/nodal vs. systemic disease). Several studies have investigated the impact of PSMA ligand PET/CT on patient management and therapy. Most studies have focused on the value of PSMA ligand PET/CT in patients with BCR after curative treatment and have reported changes in therapeutic management depending on the specific clinical scenario and the extent of treatment modification (69-74). Most recently, an overall change in the therapeutic management of $75 \%$ of 131 patients after primary treatment was shown (69). Similar results were found in a smaller cohort of 45 patients, resulting in a change of treatment in 19 of 45 patients $(42.2 \%)$, including extension of radiotherapy field or administration of dose 
escalation subsequent to local recurrence. In 2 of 19 patients, salvage radiotherapy was replaced by systemic treatment due to multiple metastatic lesions (70). In a well-defined patient cohort before salvage radiotherapy, a major management change in 20 of 70 patients $(28.6 \%)$ with a PSA level of less than $1 \mathrm{ng} / \mathrm{mL}$ was demonstrated by van Leeuwen et al. (74).

In the setting of primary treatment, a small cohort of 15 patients underwent PSMA ligand PET/CT, and the imaging was found to influence clinical TNM stage in $53.3 \%$ of patients and radiotherapy plan in $33.3 \%$ (71). Combining the settings of radiotherapy planning in both primary and recurrent disease, 2 recent publications reported PSMA ligand PET/CT to have an impact on $50.8 \%$ and $53.7 \%$ of patients $(72,73)$.

PSMA ligand PET/CT may also be used to guide salvage lymph node dissection, an emerging concept that may spare some patients ADT in early BCR. With the rise of PSMA ligand $\mathrm{PET} / \mathrm{CT}$, there is increasing interest based on both the high specificity and the improved sensitivity for detection of recurrent disease. Rauscher et al. have demonstrated high specificity $(>95 \%)$ and superior sensitivity (78\%) compared with standardof-care imaging $(27 \%)$ in patients who underwent salvage lymph node dissection (75). Preliminary results showed the feasibility of radioguided surgery exploiting preoperative labeling of lymph node metastases with a $\gamma$-emitting PSMA ligand (e.g., ${ }^{111}$ In-PSMA I\&T), allowing detection and resection of even very small metastatic lesions $(76,77)$. The recent introduction of ${ }^{99 \mathrm{~m}} \mathrm{Tc}-\mathrm{PSMA} \mathrm{I} \& \mathrm{~S}$ may facilitate dissemination of this promising technique (78).

\section{PATIENT PREPARATION AND PSMA LIGAND PET/CT IMAGE ACQUISITION}

\section{Patient Preparation}

Patients should be well hydrated before the study and during the uptake time (e.g., $500 \mathrm{~mL}$ of water orally during a 2-h period before acquisition). To reduce artifacts due to high tracer activity in the urinary system (potentially resulting in halo artifacts and false-positive findings), it is beneficial to coinject furosemide at the time of tracer injection and to have the patient empty the bladder immediately before image acquisition (79). Rectal filling with a negative contrast agent $(100-150 \mathrm{~mL})$ is optional to improve anatomic delineation of the rectum and differentiation of such structures as lymph nodes and seminal vesicles from adjacent structures.

\section{Image Acquisition}

${ }^{68} \mathrm{Ga}$-labeled PSMA ligands are applied intravenously using a recommended activity of $2 \mathrm{MBq}$ per kilogram of body weight. ${ }^{68} \mathrm{Ga}$-PSMA-11 PET/CT is routinely conducted $1 \mathrm{~h}$ after injection according to its first described clinical set-up (27). However, the same article already demonstrated that late imaging conducted at $3 \mathrm{~h}$ after injection shows most PC lesions with higher contrast because of an ongoing decrease in background signal and increase in tracer uptake. Recently, one study demonstrated that the higher uptake and contrast of PC lesions in scans at $3 \mathrm{~h}$ after injection result in a higher number of lesions detected by ${ }^{68} \mathrm{Ga}$-PSMA-11 PET/CT and a higher number of patients with an overall positive PET result (80). In contrast, most recently, another large retrospective study showed no clear advantage of delayed imaging (81). However, delayed imaging might be considered in cases of equivocal findings or in the context of low PSA levels. Imaging with ${ }^{18} \mathrm{~F}$-labeled agents has been described at $60 \mathrm{~min}$ after injection and
120 min after injection, with preliminary evidence indicating an improvement in lesion detection with later-time-point imaging $(32,82)$.

Depending on previous imaging, either a low-dose or a diagnostic CT scan with or without intravenous contrast agent is performed. The PET acquisition should be performed in 3-dimensional mode with an acquisition time of 3-4 min per bed position. Technical details on correction of emission data, image reconstruction, and postprocessing for ${ }^{68} \mathrm{Ga}$-labeled PSMA ligands have been recently published (79).

\section{PRACTICAL ISSUES}

\section{Image Display and Reading}

Hybrid PET/CT image review is recommended on a dedicated postprocessing workstation allowing parallel visualization of PET, CT, and fused PET/CT images in the axial, coronal, and sagittal planes as well as maximum-intensity projections (3-dimensional cine mode). PET and CT should be linked at the same table position to help localize PET-positive findings. For PET interpretation, both uncorrected and attenuation-corrected images need to be assessed to identify artifacts (e.g., from contrast agents, metal implants, or patient motion). Further, dynamic variation of SUV threshold by changing display windowing is necessary to adjust such variables as the uptake of PSMA ligands in or adjacent to organs with high background uptake, such as the kidneys, ureter, or urinary bladder. Otherwise, findings such as local recurrence near the urinary bladder might be missed. Semiquantitative information on suspected lesions $\left(\mathrm{SUV}_{\text {mean/max }}\right)$ can be derived on all slices of the attenuation-corrected PET study using a 3-dimensional volume of interest. Notably, there currently are no stringently defined SUV thresholds that reliably aid in differentiation between benign and malignant lesions. Diagnostic contrast-enhanced CT should be evaluated separately according to established radiologic criteria on a dedicated postprocessing workstation.

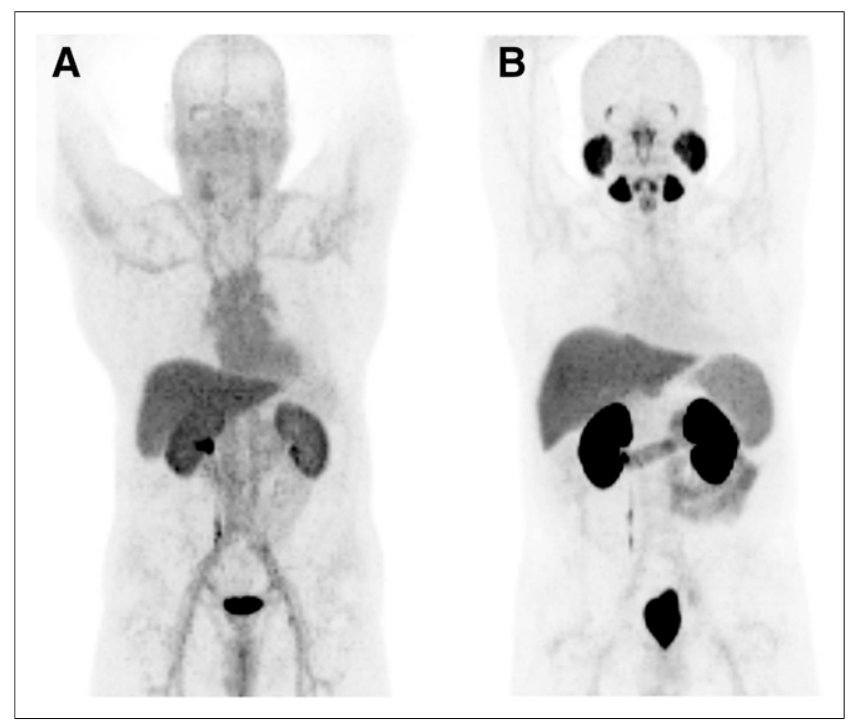

FIGURE 3. Maximum-intensity-protection images (acquired with ${ }^{18} \mathrm{~F}-$ DCFBC $[\mathrm{A}]$ and $\left.{ }^{18} \mathrm{~F}-\mathrm{DCFP} \mathrm{L}[\mathrm{B}]\right)$ displaying typical PSMA ligand biodistribution. Physiologic accumulation is seen in lacrimal and salivary glands, nasal mucosa, liver, spleen, bowel, kidneys, ureter on right side, and bladder. 


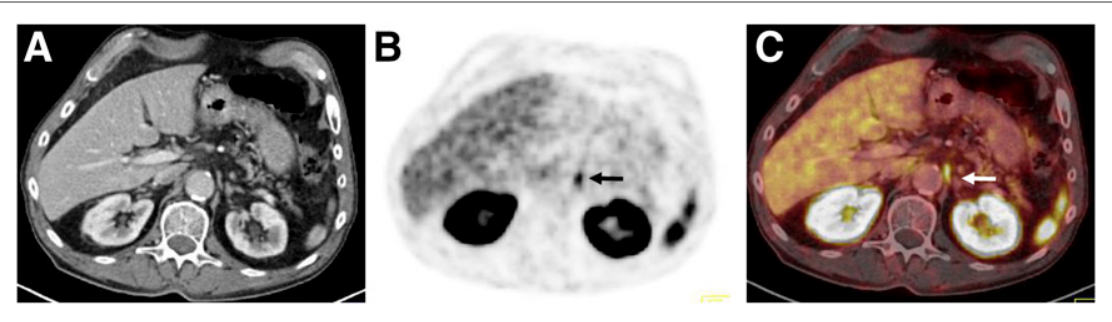

FIGURE 4. Transaxial CT (A), PET (B), and fused PET/CT (C) ${ }^{68} \mathrm{Ga}-\mathrm{PSMA}$ ligand scans demonstrating moderate, focal PSMA ligand uptake (arrows) in comma-shaped soft-tissue structure between left adrenal gland and aorta, indicating normal variant uptake in celiac ganglion.

\section{Physiologic PSMA Uptake and Variants}

All low-molecular-weight PSMA ligands for PET imaging demonstrate typical physiologic PSMA ligand uptake in the lacrimal glands, parotid glands, submandibular glands, liver, spleen, small intestine, kidneys, and colon (Fig. 3). Notably, PSMA ligand uptake in the salivary gland is not definitively proven to be related to PSMA expression in the tissue. In addition, PSMA is synonymous with $\mathrm{N}$-acetyl-L-aspartyl-L-glutamate peptidase I, which is an enzyme expressed in human brain tissue and has a role in regulating glutamate concentration.

All ${ }^{68} \mathrm{Ga}$ - and ${ }^{18} \mathrm{~F}$-labeled PSMA ligands are excreted via the kidneys, with subsequent high radiotracer uptake in the kidneys and the collected urine (16). Limited preliminary data indicate that ${ }^{18}$ F-PSMA 1007 might have reduced urinary clearance within the first $2 \mathrm{~h}$ after injection, potentially allowing for improved assessment of the prostate within this time window (33).

\section{Pathologic PSMA Uptake Related to PC and Metastases}

The excellent specificity of PSMA ligands, especially for lymph node metastases, was demonstrated in several studies $(21,39,46,83-85)$. Therefore, any focal uptake of the PSMA ligand higher than the surrounding background uptake in morphologically visible lesions and not associated with physiologic uptake should be considered suggestive.

The pathologic uptake should be reported as low, moderate, or intense by comparison to the background uptake-for example, liver or spleen-as recently described (86). Besides local involvement (primary tumor vs. local recurrence), the typical metastatic pattern is primarily the regional pelvic lymph nodes. This is often followed by distant lymph nodes (above the aortic bifurcation) and bone metastases. In advanced disease, PC can even spread to the liver, lungs, or other visceral organs.

\section{Limitations and Pitfalls in Clinical Interpretation}

It is well known that the neovasculature of many solid tumors can also expresses PSMA (22). Accordingly, there is increasing evidence that PSMA ligand uptake is not exclusively specific for PC. A large number of case series and reports describe increased PET signal in benign lesions (e.g., neurogenic tissue, Paget disease, thyroid adenoma, granulomatous disease, and adrenal adenoma) as well as in malignant diseases (e.g., renal cell carcinoma, lung cancer, glioblastoma, hepatocellular carcinoma, and thyroid cancer) (87-95). Supplemental Table 1 (supplemental materials are available at http://jnm.snmjournals.org) summarizes the current evidence in the literature. Because many of these potential pitfalls can be solved in clinical context or by adding further imaging, increased PSMA ligand uptake in ganglia is the most common pitfall (Fig. 4). Their sites (especially sacral and celiac) are near the typical locations of lymph node metastases. Thus, knowledge of the CT characteristics (e.g., size, shape, and specific location) is crucial for reliable differentiation. In a recent investigation, at least one celiac ganglion with increased PSMA ligand uptake mimicking retroperitoneal lymph node disease was found in $89 \%$ of patients undergoing ${ }^{68}$ Ga-PSMA-11 PET/ CT examinations (96). Another important limitation is the absence of PSMA overexpression in the primary tumor or its metastases in up to $10 \%$ of patients with primary PC or (as mentioned above) decreased PSMA expression in advanced disease (21). Side-by-side interpretation of the diagnostic CT scan as part of the PSMA ligand PET/CT examination is important. An example of a patient with a PSMA-positive rib fracture can be seen in Figure 5.

\section{Current Regulatory Status for PSMA Ligands}

Currently, PSMA ligands are not approved for clinical use in any country. In many European countries (especially Germany and Austria) the use of nonapproved agents for PET imaging is possible within certain limitations. For this use, the number of sites offering PSMA ligands is currently increasing because many institutions are evaluating PSMA ligands in prospective trials for either staging or restaging of PC. Most of the protocols for ${ }^{68} \mathrm{Ga}-$ PSMA-11 are harmonized under a multicentric approach headed by the Clinical Trials Network of the Society of Nuclear Medicine and Molecular Imaging. This harmonization is intended to trigger a new drug application and potential Food and Drug Administration approval. In addition, ${ }^{18} \mathrm{~F}$-DCFPyL is currently in multicenter phase II/III trials. Finally, an increasing number of clinical guidelines adopt the use of PSMA ligand PET, especially for BCR.
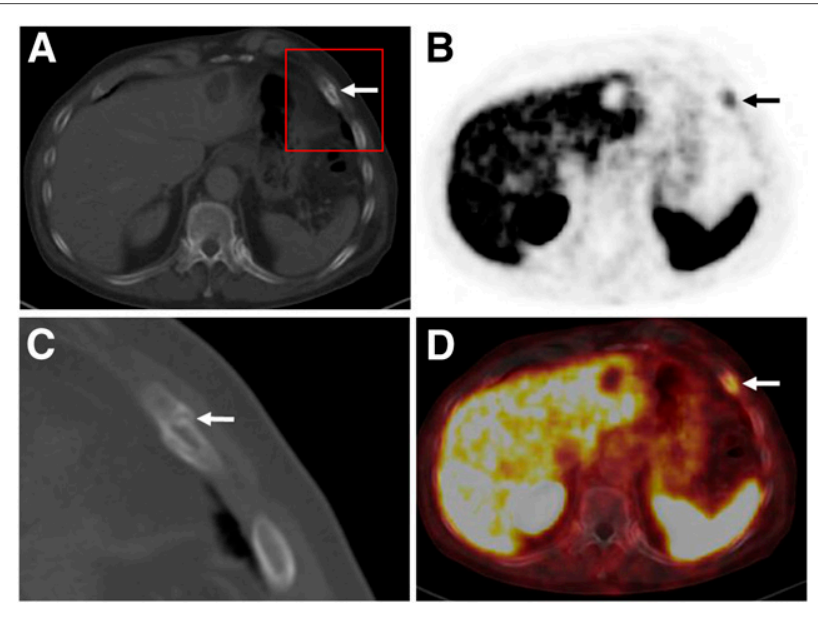

FIGURE 5. ${ }^{68} \mathrm{Ga}-\mathrm{PSMA}$ ligand PET/CT scan exhibiting moderate, focal PSMA ligand uptake in left rib on transaxial PET (B) and fused PET/CT (D) images. (A and C) Corresponding CT images confirm minimally displaced fracture of rib. 


\section{CONCLUSION}

PSMA ligand PET/CT has become a clinically accepted technique for PC imaging worldwide and provides high diagnostic efficacy in recurrent PC as well as in staging of highrisk PC. Evidence is emerging that PSMA ligand PET/CT substantially influences treatment decisions by detection of sites of recurrence and nodal or distant metastases that are often occult on standard-of-care imaging. Intraprostatic tumor localization, therapy stratification, and treatment monitoring of advanced disease are potential future indications. Standardized criteria for image interpretation of PSMA ligand PET are evolving, facilitating its use in clinical practice. Several prospective trials are under way to support final market approval and reimbursement.

\section{REFERENCES}

1. Torre LA, Siegel RL, Ward EM, Jemal A. Global cancer incidence and mortality rates and trends: an update. Cancer Epidemiol Biomarkers Prev. 2016;25:16-27.

2. Heesakkers RA, Hovels AM, Jager GJ, et al. MRI with a lymph-node-specific contrast agent as an alternative to CT scan and lymph-node dissection in patients with prostate cancer: a prospective multicohort study. Lancet Oncol. 2008;9: $850-856$.

3. Hövels AM, Heesakkers RA, Adang EM, et al. The diagnostic accuracy of CT and MRI in the staging of pelvic lymph nodes in patients with prostate cancer: a meta-analysis. Clin Radiol. 2008;63:387-395.

4. Abuzallouf S, Dayes I, Lukka H. Baseline staging of newly diagnosed prostate cancer: a summary of the literature. J Urol. 2004;171:2122-2127.

5. Turkbey B, Brown AM, Sankineni S, Wood BJ, Pinto PA, Choyke PL. Multiparametric prostate magnetic resonance imaging in the evaluation of prostate cancer. CA Cancer J Clin. 2016;66:326-336.

6. Filson CP, Natarajan S, Margolis DJ, et al. Prostate cancer detection with magnetic resonance-ultrasound fusion biopsy: The role of systematic and targeted biopsies. Cancer. 2016;122:884-892.

7. Schöder H, Herrmann K, Gonen M, et al. $2-\left[{ }^{18} \mathrm{~F}\right]$ fluoro-2-deoxyglucose positron emission tomography for the detection of disease in patients with prostatespecific antigen relapse after radical prostatectomy. Clin Cancer Res. 2005;11: 4761-4769.

8. Jadvar H. Imaging evaluation of prostate cancer with ${ }^{18} \mathrm{~F}$-fluorodeoxyglucose PET/CT: utility and limitations. Eur J Nucl Med Mol Imaging. 2013;40(suppl 1):S5-S10.

9. Souvatzoglou M, Weirich G, Schwarzenboeck S, et al. The sensitivity of $\left[{ }^{11} \mathrm{C}\right]$ choline PET/CT to localize prostate cancer depends on the tumor configuration. Clin Cancer Res. 2011;17:3751-3759.

10. Evangelista L, Guttilla A, Zattoni F, Muzzio PC, Zattoni F. Utility of choline positron emission tomography/computed tomography for lymph node involvement identification in intermediate- to high-risk prostate cancer: a systematic literature review and meta-analysis. Eur Urol. 2013;63:1040-1048.

11. Fanti S, Minozzi S, Castellucci P, et al. PET/CT with ${ }^{11} \mathrm{C}$-choline for evaluation of prostate cancer patients with biochemical recurrence: meta-analysis and critical review of available data. Eur J Nucl Med Mol Imaging. 2016;43:55-69.

12. Buchegger F, Garibotto V, Zilli T, et al. First imaging results of an intraindividual comparison of ${ }^{11} \mathrm{C}$-acetate and ${ }^{18} \mathrm{~F}$-fluorocholine PET/CT in patients with prostate cancer at early biochemical first or second relapse after prostatectomy or radiotherapy. Eur J Nucl Med Mol Imaging. 2014;41:68-78.

13. Nanni C, Schiavina R, Boschi S, et al. Comparison of ${ }^{18} \mathrm{~F}-\mathrm{FACBC}$ and ${ }^{11} \mathrm{C}-$ choline PET/CT in patients with radically treated prostate cancer and biochemical relapse: preliminary results. Eur J Nucl Med Mol Imaging. 2013;40(suppl 1): S11-S17.

14. Nanni C, Schiavina R, Brunocilla E, et al. ${ }^{18} \mathrm{~F}-\mathrm{FACBC}$ compared with ${ }^{11} \mathrm{C}$ choline PET/CT in patients with biochemical relapse after radical prostatectomy: a prospective study in 28 patients. Clin Genitourin Cancer. 2014;12:106-110.

15. Nanni C, Schiavina R, Brunocilla E, et al. ${ }^{18} \mathrm{~F}$-fluciclovine PET/CT for the detection of prostate cancer relapse: a comparison to ${ }^{11} \mathrm{C}$-choline PET/CT. Clin Nucl Med. 2015;40:e386-e391.

16. Silver DA, Pellicer I, Fair WR, Heston WD, Cordon-Cardo C. Prostate-specific membrane antigen expression in normal and malignant human tissues. Clin Cancer Res. 1997;3:81-85.
17. Mannweiler S, Amersdorfer P, Trajanoski S, Terrett JA, King D, Mehes G. Heterogeneity of prostate-specific membrane antigen (PSMA) expression in prostate carcinoma with distant metastasis. Path Oncol Res. 2009;15:167172.

18. Bostwick DG, Pacelli A, Blute M, Roche P, Murphy GP. Prostate specific membrane antigen expression in prostatic intraepithelial neoplasia and adenocarcinoma: a study of 184 cases. Cancer. 1998;82:2256-2261.

19. Troyer JK, Beckett ML, Wright GL. Detection and characterization of the prostate-specific membrane antigen (PSMA) in tissue extracts and body fluids. Int J Cancer. 1995;62:552-558.

20. Budäus L, Leyh-Bannurah SR, Salomon G, et al. Initial experience of ${ }^{68} \mathrm{Ga}-$ PSMA PET/CT imaging in high-risk prostate cancer patients prior to radical prostatectomy. Eur Urol. 2016;69:393-396.

21. Maurer T, Gschwend JE, Rauscher I, et al. Diagnostic efficacy of ${ }^{68}$ galliumPSMA positron emission tomography compared to conventional imaging for lymph node staging of 130 consecutive patients with intermediate to high risk prostate cancer. J Urol. 2016;195:1436-1443.

22. Chang SS. Overview of prostate-specific membrane antigen. Rev Urol. 2004;6 (suppl 10):S13-S18.

23. Bander NH. Technology insight: monoclonal antibody imaging of prostate cancer. Nat Clin Pract Urol. 2006;3:216-225.

24. Rajasekaran SA, Anilkumar G, Oshima E, et al. A novel cytoplasmic tail MXXXL motif mediates the internalization of prostate-specific membrane antigen. Mol Biol Cell. 2003;14:4835-4845.

25. Ghosh A, Heston WDW. Tumor target prostate specific membrane antigen (PSMA) and its regulation in prostate cancer. J Cell Biochem. 2004;91:528-539.

26. Commandeur LC, Parsons JR. Degradation of halogenated aromatic compounds. Biodegradation. 1990;1:207-220.

27. Afshar-Oromieh A, Malcher A, Eder M, et al. PET imaging with a $\left[{ }^{68} \mathrm{Ga}\right]$ gallium-labelled PSMA ligand for the diagnosis of prostate cancer: biodistribution in humans and first evaluation of tumour lesions. Eur J Nucl Med Mol Imaging. 2013;40:486-495.

28. Eder M, Schafer M, Bauder-Wust U, et al. ${ }^{68} \mathrm{Ga}$-complex lipophilicity and the targeting property of a urea-based PSMA inhibitor for PET imaging. Bioconjug Chem. 2012;23:688-697.

29. Weineisen M, Schottelius M, Simecek J, et al. ${ }^{68} \mathrm{Ga}$ - and ${ }^{177}$ Lu-labeled PSMA I\&T: optimization of a PSMA-targeted theranostic concept and first proof-ofconcept human studies. J Nucl Med. 2015;56:1169-1176.

30. Cho SY, Gage KL, Mease RC, et al. Biodistribution, tumor detection, and radiation dosimetry of ${ }^{18} \mathrm{~F}$-DCFBC, a low-molecular-weight inhibitor of prostatespecific membrane antigen, in patients with metastatic prostate cancer. $J \mathrm{Nucl}$ Med. 2012;53:1883-1891.

31. Rowe SP, Gage KL, Faraj SF, et al. ${ }^{18}$ F-DCFBC PET/CT for PSMA-based detection and characterization of primary prostate cancer. J Nucl Med. 2015;56: 1003-1010.

32. Rowe SP, Macura KJ, Mena E, et al. PSMA-based $\left[{ }^{18} \mathrm{~F}\right] \mathrm{DCFPyL}$ PET/CT is superior to conventional imaging for lesion detection in patients with metastatic prostate cancer. Mol Imaging Biol. 2016;18:411-419.

33. Giesel FL, Hadaschik B, Cardinale J, et al. F-18 labelled PSMA-1007: biodistribution, radiation dosimetry and histopathological validation of tumor lesions in prostate cancer patients. Eur J Nucl Med Mol Imaging. 2017;44:678-688.

34. Eiber MFW, Rowe SP, Calais J, et al. PSMA ligands for imaging and therapy. J Nucl Med. In press.

35. Afshar-Oromieh A, Zechmann CM, Malcher A, et al. Comparison of PET imaging with a ${ }^{68} \mathrm{Ga}$-labelled PSMA ligand and ${ }^{18} \mathrm{~F}$-choline-based PET/CT for the diagnosis of recurrent prostate cancer. Eur J Nucl Med Mol Imaging. 2014;41: 11-20.

36. Morigi JJ, Stricker PD, van Leeuwen PJ, et al. Prospective comparison of ${ }^{18} \mathrm{~F}$ fluoromethylcholine versus ${ }^{68} \mathrm{Ga}$-PSMA PET/CT in prostate cancer patients who have rising PSA after curative treatment and are being considered for targeted therapy. J Nucl Med. 2015;56:1185-1190.

37. Schwenck J, Rempp H, Reischl G, et al. Comparison of ${ }^{68} \mathrm{Ga}$-labelled PSMA-11 and ${ }^{11} \mathrm{C}$-choline in the detection of prostate cancer metastases by PET/CT. Eur J Nucl Med Mol Imaging. 2017;44:92-101.

38. Afshar-Oromieh A, Avtzi E, Giesel FL, et al. The diagnostic value of PET/CT imaging with the ${ }^{68} \mathrm{Ga}$-labelled PSMA ligand HBED-CC in the diagnosis of recurrent prostate cancer. Eur J Nucl Med Mol Imaging. 2015;42:197-209.

39. Eiber M, Maurer T, Souvatzoglou M, et al. Evaluation of hybrid ${ }^{68} \mathrm{Ga}-\mathrm{PSMA}$ ligand $\mathrm{PET} / \mathrm{CT}$ in 248 patients with biochemical recurrence after radical prostatectomy. J Nucl Med. 2015;56:668-674.

40. Afshar-Oromieh A, Holland-Letz T, Giesel FL, et al. Diagnostic performance of ${ }^{68} \mathrm{Ga}$-PSMA-11 (HBED-CC) PET/CT in patients with recurrent prostate cancer: evaluation in 1007 patients. Eur J Nucl Med Mol Imaging. 2017;44: 1258-1268. 
41. Meredith G, Wong D, Yaxley J, et al. The use of ${ }^{68}$ Ga-PSMA PET CT in men with biochemical recurrence after definitive treatment of acinar prostate cancer. BJU Int. 2016;118(suppl 3):49-55.

42. Perera M, Papa N, Christidis D, et al. Sensitivity, specificity, and predictors of positive ${ }^{68} \mathrm{Ga}$-prostate-specific membrane antigen positron emission tomography in advanced prostate cancer: a systematic review and meta-analysis. Eur Urol. 2016;70:926-937.

43. Rowe SP, Macura KJ, Ciarallo A, et al. Comparison of prostate-specific membrane antigen-based ${ }^{18} \mathrm{~F}$-DCFBC PET/CT to conventional imaging modalities for detection of hormone-naive and castration-resistant metastatic prostate cancer. J Nucl Med. 2016;57:46-53.

44. Dietlein M, Kobe C, Kuhnert G, et al. Comparison of $\left[{ }^{18} \mathrm{~F}\right] \mathrm{DCFPyL}$ and $\left[{ }^{68} \mathrm{Ga}\right]$ Ga-PSMA-HBED-CC for PSMA-PET imaging in patients with relapsed prostate cancer. Mol Imaging Biol. 2015;17:575-584.

45. Dietlein F, Kobe C, Neubauer S, et al. PSA-stratified performance of ${ }^{18} \mathrm{~F}$ - and ${ }^{68} \mathrm{Ga}$-PSMA PET in patients with biochemical recurrence of prostate cancer. J Nucl Med. 2017;58:947-958.

46. Herlemann A, Wenter V, Kretschmer A, et al. ${ }^{68} \mathrm{Ga}$-PSMA positron emission tomography/computed tomography provides accurate staging of lymph node regions prior to lymph node dissection in patients with prostate cancer. Eur Urol. 2016;70:553-557.

47. Hijazi S, Meller B, Leitsmann C, et al. Pelvic lymph node dissection for nodal oligometastatic prostate cancer detected by ${ }^{68} \mathrm{Ga}$-PSMA-positron emission tomography/computerized tomography. Prostate. 2015;75:1934-1940.

48. Pyka T, Okamoto S, Dahlbender M, et al. Comparison of bone scintigraphy and ${ }^{68} \mathrm{Ga}$-PSMA PET for skeletal staging in prostate cancer. Eur J Nucl Med Mol Imaging. 2016;43:2114-2121.

49. van Leeuwen PJ, Emmett L, Ho B, et al. Prospective evaluation of ${ }^{68}$ galliumprostate-specific membrane antigen positron emission tomography/computerized tomography for preoperative lymph node staging in prostate cancer. BJU Int. 2017;119:209-215.

50. Fendler WP, Schmidt DF, Wenter V, et al. ${ }^{68}$ Ga-PSMA PET/CT detects the location and extent of primary prostate cancer. J Nucl Med. 2016;57:17201725 .

51. Rahbar K, Weckesser M, Huss S, et al. Correlation of intraprostatic tumor extent with ${ }^{68} \mathrm{Ga}$-PSMA distribution in patients with prostate cancer. J Nucl Med. 2016;57:563-567.

52. Zamboglou C, Drendel V, Jilg CA, et al. Comparison of ${ }^{68} \mathrm{Ga}-\mathrm{HBED}-\mathrm{CC}$ PSMA$\mathrm{PET} / \mathrm{CT}$ and multiparametric MRI for gross tumour volume detection in patients with primary prostate cancer based on slice by slice comparison with histopathology. Theranostics. 2017;7:228-237.

53. Eiber M, Weirich G, Holzapfel K, et al. Simultaneous ${ }^{68}$ Ga-PSMA HBED-CC PET/MRI improves the localization of primary prostate cancer. Eur Urol. 2016;70:829-836.

54. Ceci F, Castellucci P, Nanni C, Fanti S. PET/CT imaging for evaluating response to therapy in castration-resistant prostate cancer. Eur J Nucl Med Mol Imaging. 2016;43:2103-2104.

55. Schwarzenböck SM, Eiber M, Kundt G, et al. Prospective evaluation of $\left[{ }^{11} \mathrm{C}\right]$ choline PET/CT in therapy response assessment of standardized docetaxel firstline chemotherapy in patients with advanced castration refractory prostate cancer. Eur J Nucl Med Mol Imaging. 2016;43:2105-2113.

56. Hillier SM, Kern AM, Maresca KP, et al. ${ }^{123}$ I-MIP-1072, a small-molecule inhibitor of prostate-specific membrane antigen, is effective at monitoring tumor response to taxane therapy. J Nucl Med. 2011;52:1087-1093.

57. Wright GL Jr, Grob BM, Haley C, et al. Upregulation of prostate-specific membrane antigen after androgen-deprivation therapy. Urology. 1996;48:326334.

58. Murphy G, Ragde H, Kenny G, et al. Comparison of prostate specific membrane antigen, and prostate specific antigen levels in prostatic cancer patients. Anticancer Res. 1995;15:1473-1479.

59. Liu T, Wu LY, Fulton MD, Johnson JM, Berkman CE. Prolonged androgen deprivation leads to downregulation of androgen receptor and prostate-specific membrane antigen in prostate cancer cells. Int J Oncol. 2012;41:20872092.

60. Meller B, Bremmer F, Sahlmann CO, et al. Alterations in androgen deprivation enhanced prostate-specific membrane antigen (PSMA) expression in prostate cancer cells as a target for diagnostics and therapy. EJNMMI Res. 2015;5:66.

61. Ahmadzadehfar H, Schlenkhoff CD, Rogenhofer S, Yordanova A, Essler M. ${ }^{68} \mathrm{Ga}$-PSMA-11 PET represents the tumoricidal effect of ${ }^{223} \mathrm{Ra}$ in a patient with castrate-resistant metastatic prostate cancer. Clin Nucl Med. 2016;41: 695-696.

62. Ahmadzadehfar H, Eppard E, Kurpig S, et al. Therapeutic response and side effects of repeated radioligand therapy with ${ }^{177}$ Lu-PSMA-DKFZ-617 of castrate-resistant metastatic prostate cancer. Oncotarget. 2016;7:1247712488.

63. Baum RP, Kulkarni HR, Schuchardt C, et al. ${ }^{177}$ Lu-labeled prostate-specific membrane antigen radioligand therapy of metastatic castration-resistant prostate cancer: safety and efficacy. J Nucl Med. 2016;57:1006-1013.

64. Heck MM, Retz M, D’Alessandria C, et al. Systemic radioligand therapy with ${ }^{177} \mathrm{Lu}$ labeled prostate specific membrane antigen ligand for imaging and therapy in patients with metastatic castration resistant prostate cancer. J Urol. 2016; 196:382-391.

65. Parimi V, Goyal R, Poropatich K, Yang XJ. Neuroendocrine differentiation of prostate cancer: a review. Am J Clin Exp Urol. 2014;2:273-285.

66. Yuan TC, Veeramani S, Lin MF. Neuroendocrine-like prostate cancer cells: neuroendocrine transdifferentiation of prostate adenocarcinoma cells. Endocr Relat Cancer. 2007;14:531-547.

67. Afshar-Oromieh A, Babich JW, Kratochwil C, et al. The rise of PSMA ligands for diagnosis and therapy of prostate cancer. J Nucl Med. 2016;57(suppl):79S$89 \mathrm{~S}$.

68. Rowe SP, Drzezga A, Neumaier B, et al. Prostate-specific membrane antigentargeted radiohalogenated PET and therapeutic agents for prostate cancer. J Nucl Med. 2016;57(suppl):90S-96S.

69. Albisinni S, Artigas C, Aoun F, et al. Clinical impact of ${ }^{68} \mathrm{Ga}$-prostate-specific membrane antigen (PSMA) positron emission tomography/computed tomography $(\mathrm{PET} / \mathrm{CT})$ in patients with prostate cancer with rising prostate-specific antigen after treatment with curative intent: preliminary analysis of a multidisciplinary approach. BJU Int. December 15, 2016 [Epub ahead of print].

70. Bluemel C, Linke F, Herrmann K, et al. Impact of ${ }^{68} \mathrm{Ga}$-PSMA PET/CT on salvage radiotherapy planning in patients with prostate cancer and persisting PSA values or biochemical relapse after prostatectomy. EJNMMI Res. 2016;6:78.

71. Dewes S, Schiller K, Sauter K, et al. Integration of ${ }^{68}$ Ga-PSMA-PET imaging in planning of primary definitive radiotherapy in prostate cancer: a retrospective study. Radiat Oncol. 2016;11:73.

72. Shakespeare TP. Effect of prostate-specific membrane antigen positron emission tomography on the decision-making of radiation oncologists. Radiat Oncol. 2015;10:233.

73. Sterzing F, Kratochwil C, Fiedler H, et al. ${ }^{68}$ Ga-PSMA-11 PET/CT: a new technique with high potential for the radiotherapeutic management of prostate cancer patients. Eur J Nucl Med Mol Imaging. 2016;43:34-41.

74. van Leeuwen PJ, Stricker P, Hruby G, et al. ${ }^{68}$ Ga-PSMA has a high detection rate of prostate cancer recurrence outside the prostatic fossa in patients being considered for salvage radiation treatment. BJU Int. 2016;117: 732-739.

75. Rauscher I, Maurer T, Beer AJ, et al. Value of ${ }^{68} \mathrm{Ga}$-PSMA HBED-CC PET for the assessment of lymph node metastases in prostate cancer patients with biochemical recurrence: comparison with histopathology after salvage lymphadenectomy. J Nucl Med. 2016;57:1713-1719.

76. Maurer T, Weirich G, Schottelius M, et al. Prostate-specific membrane antigenradioguided surgery for metastatic lymph nodes in prostate cancer. Eur Urol. 2015;68:530-534.

77. Rauscher I, Düwel C, Wirtz M, et al. Value of ${ }^{111}$ In-prostate-specific membrane antigen (PSMA)-radioguided surgery for salvage lymphadenectomy in recurrent prostate cancer: correlation with histopathology and clinical follow-up. BJU Int. 2017; 120:40-47.

78. Robu S, Schottelius M, Eiber M, et al. Preclinical evaluation and first patient application of ${ }^{99 \mathrm{~m}} \mathrm{Tc}-\mathrm{PSMA}-\mathrm{I} \& \mathrm{~S}$ for SPECT imaging and radioguided surgery in prostate cancer. J Nucl Med. 2017;58:235-242.

79. Fendler WP, Eiber M, Beheshti M, et al. ${ }^{68} \mathrm{Ga}$-PSMA PET/CT: joint EANM and SNMMI procedure guideline for prostate cancer imaging: version 1.0. Eur J Nucl Med Mol Imaging. 2017;44:1014-1024.

80. Afshar-Oromieh A, Sattler LP, Mier W, et al. The clinical impact of additional late PET/CT imaging with ${ }^{68} \mathrm{Ga}-\mathrm{PSMA}-11$ (HBED-CC) in the diagnosis of prostate cancer. J Nucl Med. 2017;58:750-755.

81. Schmuck S, Nordlohne S, von Klot CA, et al. Comparison of standard and delayed imaging to improve the detection rate of $\left[{ }^{68} \mathrm{Ga}\right] P S M A ~ I \& T$ PET/CT in patients with biochemical recurrence or prostate-specific antigen persistence after primary therapy for prostate cancer. Eur J Nucl Med Mol Imaging. 2017;44:960-968.

82. Wondergem M, van der Zant F, Knol R, Lazarenko S, Pruim J, de Jong IJ. ${ }^{18} \mathrm{~F}$ DCFPyL PET/CT in the detection of prostate cancer at 60 and 120 minutes; detection rate, image quality, activity kinetics and biodistribution. J Nucl Med. April 27, 2017 [Epub ahead of print].

83. Afshar-Oromieh A, Hetzheim H, Kratochwil C, et al. The theranostic PSMA ligand PSMA-617 in the diagnosis of prostate cancer by PET/CT: biodistribution 
in humans, radiation dosimetry, and first evaluation of tumor lesions. J Nucl Med. 2015;56:1697-1705.

84. Pfister D, Porres D, Heidenreich A, et al. Detection of recurrent prostate cancer lesions before salvage lymphadenectomy is more accurate with ${ }^{68} \mathrm{Ga}$-PSMAHBED-CC than with ${ }^{18}$ F-fluoroethylcholine PET/CT. Eur J Nucl Med Mol Imaging. 2016;43:1410-1417.

85. Sahlmann CO, Meller B, Bouter C, et al. Biphasic ${ }^{68}$ Ga-PSMA-HBED-CC-PET/ $\mathrm{CT}$ in patients with recurrent and high-risk prostate carcinoma. Eur J Nucl Med Mol Imaging. 2016;43:898-905.

86. Rauscher I, Maurer T, Fendler WP, Sommer WH, Schwaiger M, Eiber M. ${ }^{68} \mathrm{Ga}-$ PSMA ligand PET/CT in patients with prostate cancer: how we review and report. Cancer Imaging. 2016;16:14.

87. Artigas C, Alexiou J, Garcia C, et al. Paget bone disease demonstrated on ${ }^{68} \mathrm{Ga}$-PSMA ligand PET/CT. Eur J Nucl Med Mol Imaging. 2016;43:195196.

88. Kanthan GL, Coyle L, Kneebone A, Schembri GP, Hsiao E. Follicular lymphoma showing avid uptake on ${ }^{68} \mathrm{Ga}$ PSMA-HBED-CC PET/CT. Clin Nucl Med. 2016;41:500-501.

89. Kobe C, Maintz D, Fischer T, Drzezga A, Chang DH. Prostate-specific membrane antigen PET/CT in splenic sarcoidosis. Clin Nucl Med. 2015;40:897898.
90. Pyka T, Weirich G, Einspieler I, et al. ${ }^{68}$ Ga-PSMA-HBED-CC PET for differential diagnosis of suggestive lung lesions in patients with prostate cancer. $\mathrm{J} \mathrm{Nucl}$ Med. 2016;57:367-371.

91. Rischpler C, Maurer T, Schwaiger M, Eiber M. Intense PSMA-expression using ${ }^{68} \mathrm{Ga}$-PSMA PET/CT in a paravertebral schwannoma mimicking prostate cancer metastasis. Eur J Nucl Med Mol Imaging. 2016;43:193-194.

92. Rowe SP, Gorin MA, Hammers HJ, Pomper MG, Allaf ME, Javadi MS. Detection of ${ }^{18} \mathrm{~F}-\mathrm{FDG}$ PET/CT occult lesions with ${ }^{18} \mathrm{~F}-\mathrm{DCFPyL}$ PET/CT in a patient with metastatic renal cell carcinoma. Clin Nucl Med. 2016;41:83-85.

93. Sasikumar A, Joy A, Nanabala R, Pillai MR, Thomas B, Vikraman KR. ${ }^{68} \mathrm{Ga}-$ PSMA PET/CT imaging in primary hepatocellular carcinoma. Eur J Nucl Med Mol Imaging. 2016;43:795-796.

94. Schwenck J, Tabatabai G, Skardelly M, et al. In vivo visualization of prostatespecific membrane antigen in glioblastoma. Eur J Nucl Med Mol Imaging. 2015;42:170-171

95. Verburg FA, Krohn T, Heinzel A, Mottaghy FM, Behrendt FF. First evidence of PSMA expression in differentiated thyroid cancer using $\left[{ }^{68} \mathrm{Ga}\right] \mathrm{PSMA}-\mathrm{HBED}-\mathrm{CC}$ PET/CT. Eur J Nucl Med Mol Imaging. 2015;42:1622-1623.

96. Krohn T, Verburg FA, Pufe T, et al. [ $\left.{ }^{68} \mathrm{Ga}\right]$ PSMA-HBED uptake mimicking lymph node metastasis in coeliac ganglia: an important pitfall in clinical practice. Eur J Nucl Med Mol Imaging. 2015;42:210-214. 